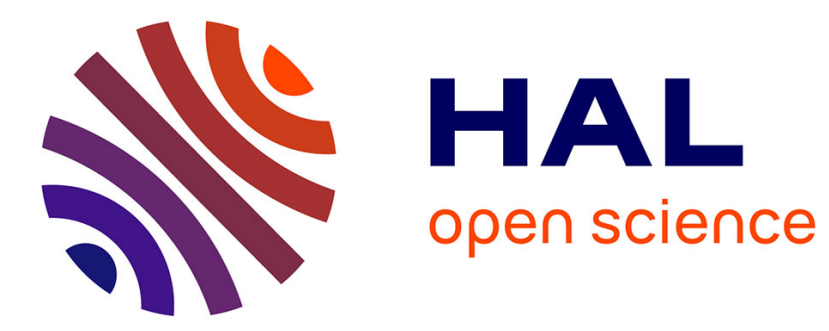

\title{
Effets géométriques dans la thermodynamique de la transition de l'état diamagnétique à l'état intermédiaire des supraconducteurs de première espèce
}

\author{
A. Fortini, E. Paumier, J. Provost, J.-P. Girard
}

\section{- To cite this version:}

A. Fortini, E. Paumier, J. Provost, J.-P. Girard. Effets géométriques dans la thermodynamique de la transition de l'état diamagnétique à l'état intermédiaire des supraconducteurs de première espèce. Revue de Physique Appliquée, 1973, 8 (4), pp.427-431. 10.1051/rphysap:0197300804042700 . jpa00243709

\author{
HAL Id: jpa-00243709 \\ https://hal.science/jpa-00243709
}

Submitted on 1 Jan 1973

HAL is a multi-disciplinary open access archive for the deposit and dissemination of scientific research documents, whether they are published or not. The documents may come from teaching and research institutions in France or abroad, or from public or private research centers.
L'archive ouverte pluridisciplinaire HAL, est destinée au dépôt et à la diffusion de documents scientifiques de niveau recherche, publiés ou non, émanant des établissements d'enseignement et de recherche français ou étrangers, des laboratoires publics ou privés. 


\title{
EFFETS GÉOMÉTRIQUES DANS LA THERMODYNAMIQUE DE LA TRANSITION DE L'ÉTAT DIAMAGNÉTIQUE A L'ÉTAT INTERMÉDIAIRE DES SUPRACONDUCTEURS DE PREMIẼRE ESPẼCE
}

\author{
A. FORTINI, E. PAUMIER, J. PROVOST, J.-P. GIRARD
}

Laboratoire de Physique du Solide de l'Université de Caen, 14032 Caen Cedex, France

\begin{abstract}
Résumé. - Nous montrons que le champ d'entrée dans l'état intermédiaire d'un disque supraconducteur de première espèce est déterminé par la condition de migration des domaines normaux vers le centre. Ce champ est supérieur à celui pour lequel l'existence de domaines au centre serait énergétiquement favorable. La persistance de l'état diamagnétique au-delà de ce dernier champ peut ainsi être interprétée par l'existence de barrières d'énergie dépendant de la forme de l'échantillon. Ce processus se trouve confirmé par l'étude expérimentale de l'aimantation de disques de plomb.
\end{abstract}

Abstract. - It is shown that the transition field into the intermediate state of a first kind superconducting disc is determined by the migration of normal domains towards the centre. This field is greater than the field for which the existence of central domains would be energetically favourable. The persistence of the diamagnetic state beyond the latter field can thus be interpreted by the existence of energy walls depending upon the shape of the sample. This process is confirmed by the experimental study of the magnetization of lead discs.

1. Introduction. - Un échantillon supraconducteur pur de forme ellipsoïdale soumis à un champ magnétique uniforme croissant à partir de zéro évolue de l'état parfaitement diamagnétique (D) à l'état normal (N) en passant par un état dit intermédiaire (I). $\mathrm{Si}$ l'on ignore la structure fine de l'état intermédiaire établie par Landau [1] on peut aisément calculer l'énergie libre et le moment magnétique en fonction du champ appliqué $H_{0}$ pour chacune des phases $\mathrm{D}$ et I [2] (Fig. 1). La transition D-I se produit pour la valeur $H_{\mathrm{DI}}=(1-v) H_{\mathrm{c}}$ du champ appliqué, $H_{\mathrm{c}}$ désignant le champ critique et $v$ le facteur démagnétisant.

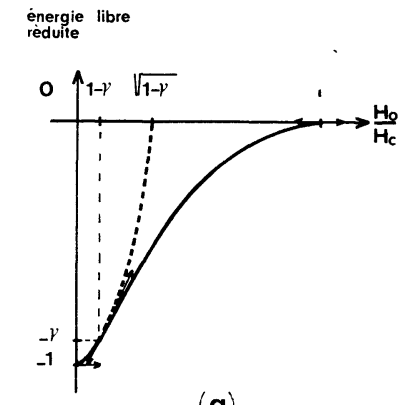

(a)

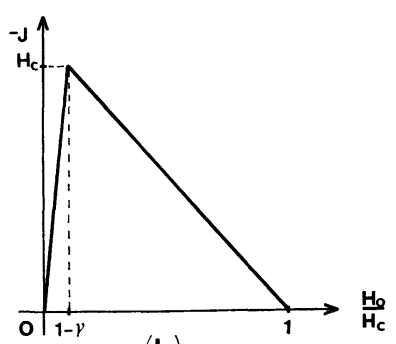

(b)
Fig. 1. - Variation de l'énergie libre réduite $(a)$ et de l'aimantation $J(b)$ en fonction du champ réduit $H_{0} / H_{\mathrm{c}}$ pour un ellipsoïde de coefficient démagnétisant $v$.
Les expériences effectuées sur des sphères ou des cylindres de grand diamètre en champ transverse [3], [4] montrent que les courbes d'aimantation sont presque parfaitement réversibles.

Lorsque la dimension de l'échantillon parallèle au champ, soit $l$, n'est plus très supérieure au paramètre d'énergie de surface $\Delta$ (l'énergie par unité de surface de paroi est $\frac{1}{2} \mu_{0} H_{\mathrm{c}}^{2} \Delta$ ), il est nécessaire de prendre en compte l'énergie des parois et le caractère discontinu de la structure de domaine dans l'énergie magnétique. Landau [1] a le premier calculé les termes supplémentaires qui en résultent dans l'énergie libre. En fait, l'énergie magnétique s'accroît par suite de la distorsion des lignes de force en bout de domaines, au voisinage des surfaces plus ou moins perpendiculaires au champ. La somme des termes supplémentaires présente un minimum qui fixe, du moins en principe, la distribution et le pas de la structure de domaines la plus favorable. On obtient en outre un déplacement du champ d'entrée dans l'état intermédiaire $H_{\mathrm{DI}}$, en gros proportionnel à une certaine puissance du rapport $\Delta / l$. Selon cette description la transition de phase doit être réversible.

Les premiers calculs de Landau ont été perfectionnés par Landau lui-même [5] qui y introduisit le «branching » ou ramification des domaines au voisi- 
nage de la surface de l'échantillon, puis par divers auteurs [2], [6] qui ont établi des formules plus ou moins élaborées pour l'énergie libre et les champs de transition.

L'accord avec les expériences effectuées soit sur des cylindres de faible diamètre en champ transverse [7], soit sur des disques [8], est assez médiocre.

A la suite de nouveaux calculs que nous avons effectués [2] en nous basant sur les idées de Landau et conduisant à un déplacement du champ de transition en $(\Delta / l)^{1 / 3}$ nous avons reconsidéré les mesures de moment magnétique sur des échantillons en forme de disques de diamètre $a=9 \mathrm{~mm}$ et d'épaisseurs $l$ comprises entre 0,2 et $1 \mathrm{~mm}$. Dans tous les cas le déplacement observé du champ de transition par rapport à la valeur «idéale » proche de $(\pi l / 2 a) H_{\mathrm{c}}$, était parfaitement reproductible, mais nettement supérieur à la valeur attendue (5 à $10 \%$ ) et s'accompagnait d'effets d'hystérésis.

Ces observations suggèrent que les effets de bout de domaine sont de faible importance et que la transition est en fait gouvernée par un mécanisme différent que nous allons maintenant analyser.

2. Thermodynamique de la transition de phase. Le développement récent des moyens d'observation directe de la structure de domaines [9 à 14] et certaines mesures soignées de moment magnétique [4], [15] ont permis d'élucider les étapes de la transformation de phase et révélé le rôle important que joue la géométrie de l'échantillon. Shoenberg [3] avait d'ailleurs signalé dès 1937 que l'apparition d'hystérésis est étroitement liée à la forme des échantillons.

Considérons un échantillon de section rectangulaire dans le plan $x \mathrm{O} z$, que pour simplifier nous supposons infini dans la direction $\mathrm{Oy}$ (Fig. 2). Le champ magnétique $H_{0}$ est appliqué selon $\mathrm{O} z$.

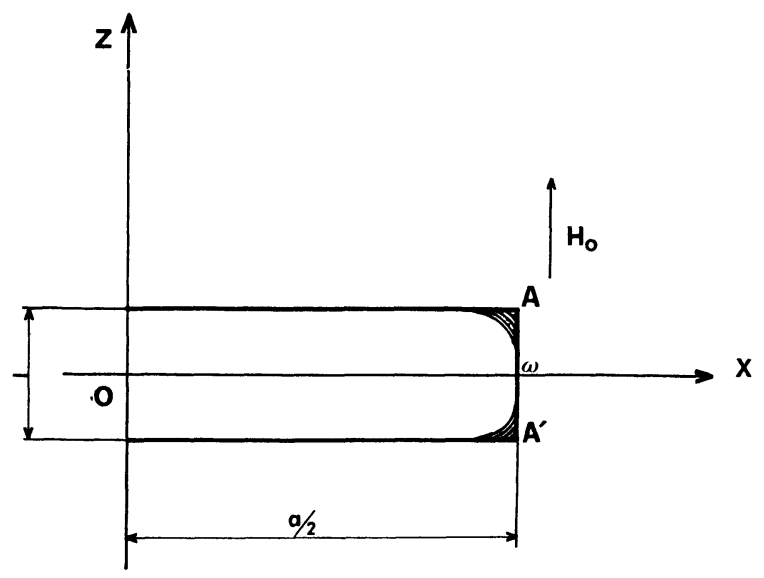

FIG. 2. - Profil d'une plaque supraconductrice par rapport aux axes de coordonnées, placée perpendiculairement au champ $H_{0}$. Les hachures dans le voisinage de $\mathrm{A}$ et $\mathrm{A}^{\prime}$ représentent la pénétration du flux.

$\mathrm{Si}$ on suppose que l'échantillon est complètement diamagnétique la distribution du champ peut être déterminée par transformation conforme. Le calcul fournit une valeur infinie pour le champ en A (effet de pointe). Il en résulte que dès que le champ $H_{0}$ est différent de zéro une structure d'état intermédiaire apparaît dans les angles, ainsi que l'ont montré les observations de Baird [13] et les mesures de Girard [15].

En assimilant cette structure d'état intermédiaire à l'état normal, il est possible d'améliorer la transformation conforme et d'obtenir une description mathématique de la distribution de champ plus proche de la réalité.

Lorsque $l \ll a$, on peut assimiler l'échantillon à un cylindre elliptique d'axes $l$ et $a$. On pourrait donc s'attendre à une entrée dans l'état intermédiaire pour un champ voisin de $\frac{l}{a} H_{\mathrm{c}}$. Or, les mesures de moment magnétique montrent que le champ de transition $H_{\mathrm{DI}}$ est très supérieur; en outre, les observations de Baird [13] et de Olafsson et Allen [12] ont montré que l'état intermédiaire commence à s'établir par migration de domaines de la périphérie vers le centre de l'échantillon, au moment où les régions intermédiaires localisées dans les angles $\mathrm{A}$ et $\mathrm{A}^{\prime}$ se rejoignent en $\omega$ dans le plan équatorial.

En nous basant sur ces observations nous avons recherché la valeur du champ appliqué pour laquelle un domaine disposé au centre de l'échantillon abaisse l'énergie libre. Or si $V_{\mathrm{d}}$ est le volume d'un domaine fictif immergé dans la matière diamagnétique et $H_{\mathrm{d}}$ le champ qui y règne, l'accroissement d'énergie libre par rapport à l'état diamagnétique est :

$$
\Delta F=\frac{1}{2} \mu_{0} V_{\mathrm{d}}\left(H_{\mathrm{c}}^{2}-H_{\mathrm{d}}^{2}\right) .
$$

Le premier terme est dû au passage à l'état normal du volume $V_{d}$ et le second à la pénétration du champ magnétique dans le même volume.

Autrement dit, l'apparition d'un domaine central serait « stabilisante" pour $H_{\mathrm{d}}=H_{\mathrm{c}}$. Il est possible de déterminer approximativement, par transformation conforme, la valeur du champ appliqué pour laquelle cette condition est réalisée. On trouve, pour un domaine de dimension $d$, avec $d \ll l \ll a$.

$$
H_{0}=H_{\mathrm{c}} \frac{l}{a}\left[1-\frac{l}{\pi a}\left(1+\log 2 \pi \frac{a}{l}\right)\right] \text {. }
$$

Cette valeur est proche de la valeur idéale donc encore nettement inférieure à la valeur observée.

D'autre part, il est possible, toujours par transformation conforme, de déterminer le champ $H_{0}$ pour lequel $H_{\omega}=H_{c}$, ce qui est la condition pour que les régions intermédiaires des angles se rejoignent en $\omega$. On trouve :

$$
H_{0}=H_{\mathrm{c}}\left(\frac{2 l}{\pi a}\right)^{1 / 2}\left[1-\frac{l}{2 \pi a} \log 2 \pi \frac{a}{l}\right] .
$$

La différence qu'on trouve ainsi entre cette dernière valeur et la valeur (2) du champ pour laquelle l'apparition du domaine au centre est stable est l'indice qu'un 
processus métastable s'établit à partir du champ idéal (2). Pour le comprendre, nous faisons d'abord appel au théorème du fluxoïde. Il est bien connu [16] que le flux magnétique à travers un contour situé à l'intérieur de la matière supraconductrice, et loin de toute paroi (à une distance supérieure à la longueur de pénétration de London), ne peut varier au cours du temps. Il en résulte que la création sur place d'un domaine est impossible et que par suite, pour apparaître au centre de l'échantillon, un domaine doit nécessairement migrer à partir de la surface.

Supposons alors que le champ ait atteint la valeur (2). L'énergie libre commence en principe à s'abaisser si un domaine existe au centre de l'échantillon, mais il ne peut y parvenir que si toutes les positions intermédiaires sont possibles, ce qui exige selon l'expression (1), que le champ y ait la valeur critique. Or lorsque $H_{0}$ prend la valeur (2) la condition $H_{\mathrm{d}}=H_{\mathrm{c}}$ est juste réalisée pour un domaine en position centrale $\mathrm{c}^{\prime} \mathrm{c}$ mais ne l'est en aucune position à droite (Fig. $3 a$ ) car le potentiel magnétique $\varphi$ décroît de $c^{\prime}$ à $\omega$ (on peut supposer $\varphi_{\omega}=0$ ).
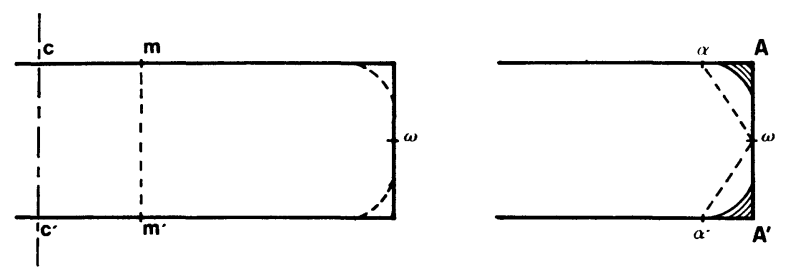

$H_{0}=\frac{1}{a} H_{c} \quad \frac{1}{a} H_{c}<H_{0}<\left(\frac{21}{\pi d}\right)^{1 / 2} H_{c} \quad H_{b}=\left(\frac{21}{\pi d}\right)^{1 / 2} H_{c}$

(a)
$H_{0} \approx\left(\frac{2 I}{\pi a}\right)^{1 / 2} H_{c}$

(b)
FIG. 3a. - Positions limites d'existence d'un domaine suivant les valeurs du champ appliqué $H_{0}$. b) Configuration limite possible à la transition.

Lorsque le champ est compris entre les valeurs (2) et (3) la condition $H_{\mathrm{d}}=H_{\mathrm{c}}$ n'est remplie que pour les domaines situés à gauche d'une certaine position limite $\mathrm{m}^{\prime} \mathrm{m}$ (Fig. 3a). Ce domaine limite est rectiligne car il correspond à la différence minimale de potentiel magnétique entre deux points symétriques $\mathrm{m}$ et $\mathrm{m}^{\prime}$ :

$$
\varphi_{\mathrm{m}^{\prime}}-\varphi_{\mathrm{m}}=2 \varphi_{\mathrm{m}^{\prime}}=l H_{\mathrm{c}} .
$$

Enfin, lorsque $H_{0}$ atteint la limite donnée par (3) le premier domaine, tangent en $\omega$, qui a pénétré dans l'échantillon peut se propager vers le centre.

Tout se passe comme si dans l'intervalle compris entre les valeurs (2) et (3) de $H_{0}$ où l'existence des domaines au centre est théoriquement stable, une « barrière d'énergie magnétique », due à la forme de l'échantillon, s'opposait à la migration. L'échantillon demeure alors réversiblement dans une phase diamagnétique (à la pénétration près dans les coins) que l'on peut considérer comme métastable dans cet intervalle.

En toute rigueur le champ de transition n'est d'ailleurs pas forcément déterminé par la condition $H_{\omega}=H_{\mathrm{c}}$ mais par la stabilité d'une configuration limite telle que $\alpha^{\prime} \omega \alpha$ (Fig. $3 b$ ) qui peut se produire pour un champ légèrement inférieur tel que :

$$
\varphi_{\alpha^{\prime}}=\alpha^{\prime} \omega \cdot H_{\mathrm{c}} .
$$

Au-delà du seuil donné par (3) l'accumulation des domaines dans la partie centrale s'accroît, comme le montrent les observations directes, et un état intermédiaire plus ou moins inhomogène s'étend progressivement à tout l'échantillon.

L'évolution de la pénétration du flux est schématisée sur la figure 4, avec les intervalles caractéristiques de variation du champ magnétique.
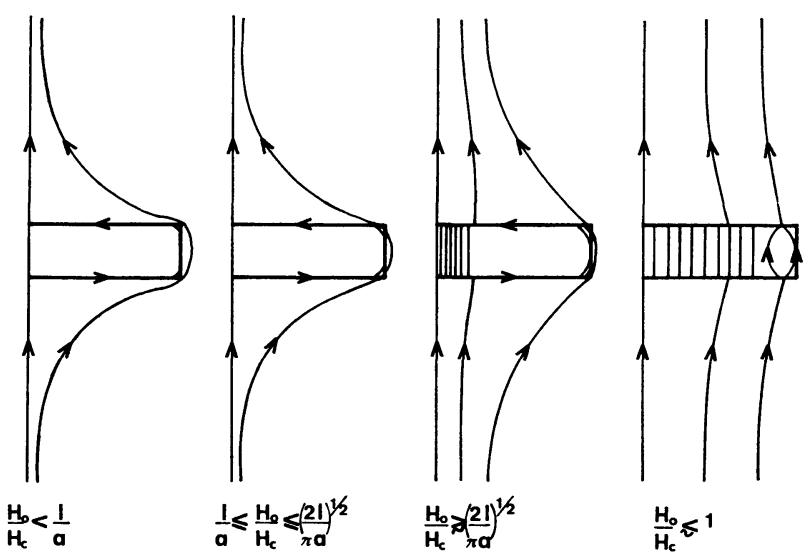

FIG. 4. - Représentation schématique de l'évolution de la pénétration du flux quand le champ appliqué $H_{0}$ croît de 0 à $H_{\text {c. }}$.

Sur la figure 5 on a représenté qualitativement l'évolution de l'énergie libre et du moment magnétique d'une plaque de volume $v$. On notera que l'aire théorique $(\mathrm{ODN})=\frac{1}{2} v H_{\mathrm{c}}^{2}$ (Fig. $\left.5 b\right)$ correspond à la transformation idéale. L'aire (DEI) correspond au comportement métastable. L'aire (EIN) peut également être due à un mécanisme métastable dans la phase intermédiaire partielle, mais aussi au mouvement irréversible des domaines dû à divers obstacles.

Finalement l'existence de barrières d'énergie rend inévitable l'apparition d'effet d'hystérésis et on comprend ainsi que ces effets soient étroitement liés à la forme de l'échantillon [3], [4], [15]. Les barrières d'énergie ne disparaissent parfaitement que dans un échantillon ellipsoïdal.

3. Vérification expérimentale. - La dépendance en $(l / a)^{1 / 2}$ du champ de transition peut être vérifiée expérimentalement. La figure 6 montre la comparaison avec la théorie de diverses mesures qui ont été publiées (à notre connaissance) et des mesures que nous avons effectuées sur le plomb à $4,2 \mathrm{~K}$.

Dans le calcul on a ignoré ici la pénétration dans les coins ce qui conduit pour $l \ll a$ à l'expression suivante du champ de transition, légèrement différente de (3)

$H_{\mathrm{DI}}=\left(\frac{4 l}{\pi a}\right)^{1 / 2}\left[1-\left(\log \frac{4 \pi a}{l}+1\right) \frac{l}{2 \pi a}\right] H_{\mathrm{c}}$. 


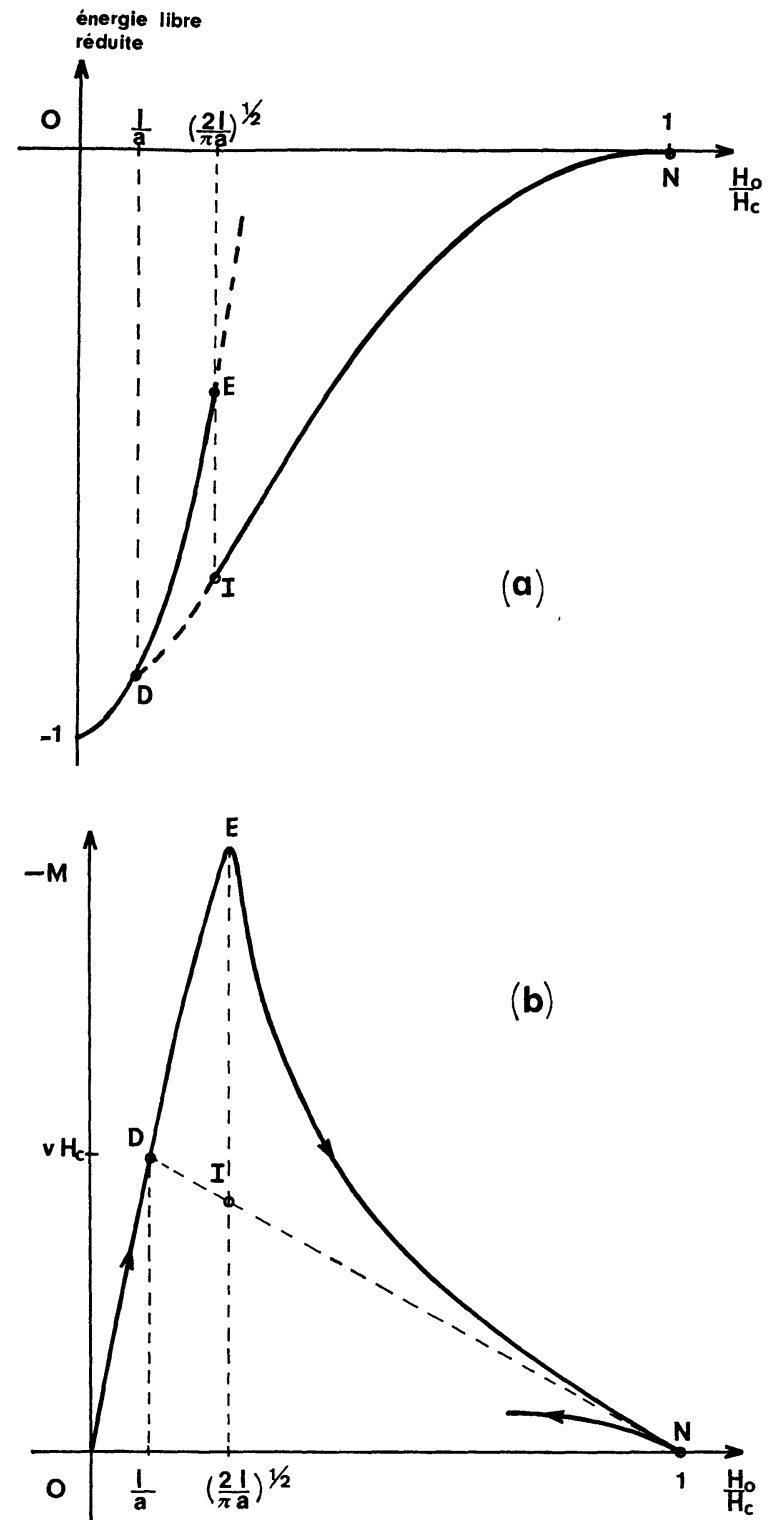

Fig. 5. - Allure de la variation de l'énergie libre réduite $(a)$ et du moment magnétique $(b)$ en fonction du champ appliqué dans le cas de la plaque de la figure 2 .

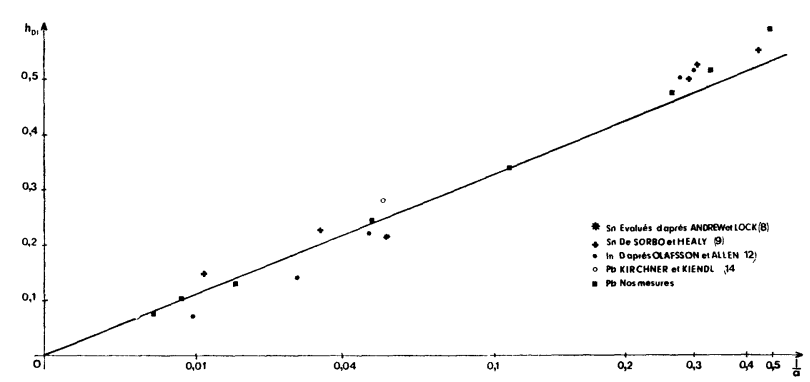

Fig. 6. - Comparaison du comportement théorique donné par (4) (courbe en trait plein) du champ de transition réduit $h_{\mathrm{DI}}=H_{\mathrm{DI}} / \mathrm{H}_{\mathrm{c}}$ en fonction du rapport des dimensions $l / a$ avec les mesures effectuées par divers auteurs.
L'accord est bon, bien que les échantillons soient des disques alors que le calcul correspond à des lames infiniment longues. Une vérification plus précise est en cours sur des lames allongées, en se basant sur l'expression plus réaliste (3).

L'existence de barrières d'énergie magnétique peut également être contrôlée expérimentalement en les supprimant de diverses façons sur une petite région de la périphérie. Un moyen particulièrement simple consiste à faire un amincissement de petite dimension qui supprime ou abaisse suffisamment la barrière à cet endroit, ce qui permet aux domaines de gagner la région centrale dès que la stabilité le permet. $\mathrm{La}$ courbe d'aimantation est profondément modifiée comme le montre la figure 7 . Le champ de transition
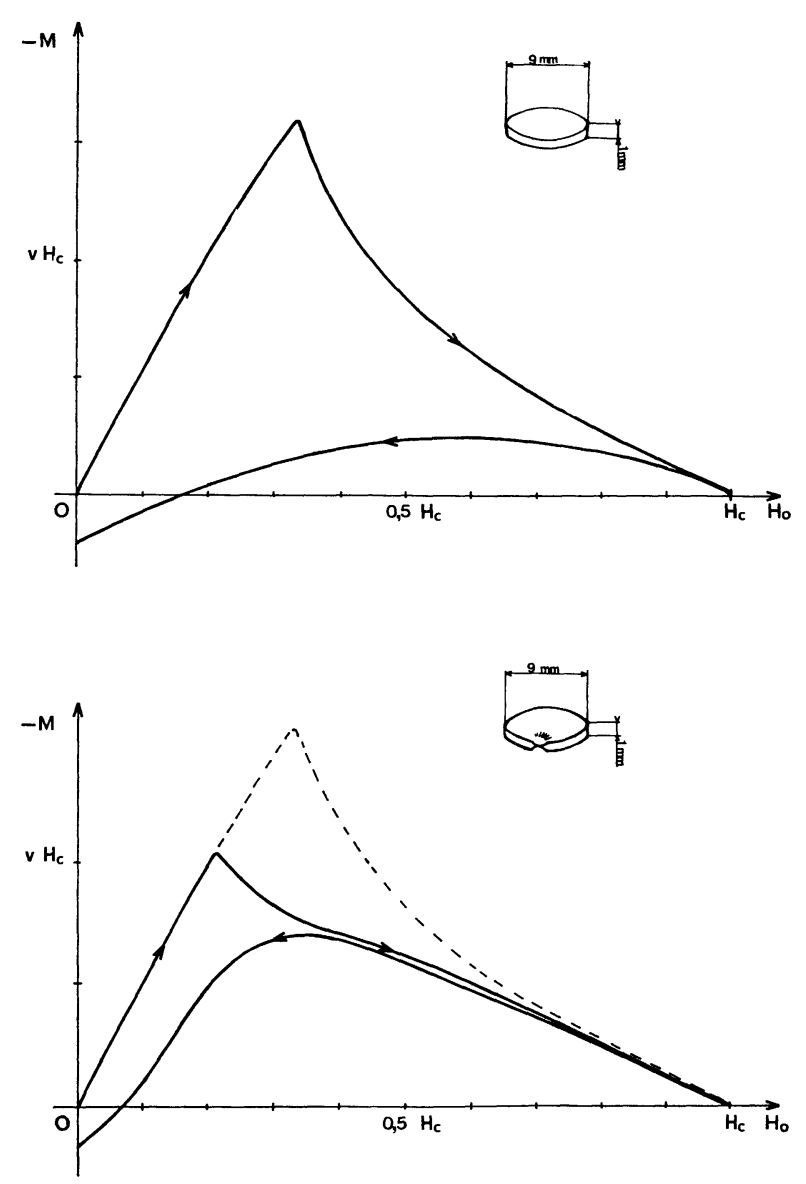

FIG. 7. - Modification de la courbe d'aimantation d'un disque de plomb sous l'effet d'un amincissement local du bord permettant la migration des domaines vers le centre.

est maintenant très proche de la valeur idéale (2) et les effets d'hystérésis sont très atténués. Ces observations confirment l'existence de la phase théorique correspondant au trajet DI de la figure 5. 


\section{Bibliographie}

[1] Landau, L. D., Phys. Z. Sowjet 11 (1937) 129.

[2] Fortini, A. and Paumier, E., Phys. Rev. B 5 (1972) 1850.

[3] Shoenberg, D., Proc. Phil. Soc. Cambridge 33 (1937) 260.

[4] Girard, J. P., Thèse de Doctorat de $3^{\mathrm{e}}$ cycle, Caen, 1972.

[5] LANDAU, L. D., J. Phys. USSR 7 (1943) 99.

[6] Andrew, E. R., Proc. R. Soc. A 194 (1948) 98.

[7] Désirant, M., Shoenberg, D., Proc. R. Soc. A 194 (1948) 63.

[8] Andrew, E. R. and Lock, J. M., Proc. Phys. Soc. A 63 (1950) 13.

[9] De Sorbo, W. and Healy, W. A., Cryogenics 4 (1964) 257.
[10] BaIrd, D. C., Can. J. Phys. 42 (1964) 1682.

[11] Haenssler, F., Rinderer, L., Helv. Phys. Acta 40 (1967) 659.

[12] Olafsson, R. and Allen, J. F., J. Phys. F, Metal Phys. 2 (1972) 123.

[13] Baird, D. C. and Wright, L. S., J. Low Temp. Phys. 8 (1972) 177

[14] Kirchner, H., Kiendl, A., Phys. Lett. A 39 (1972) 293.

[15] Girard, J. P., Paumier, E. et Fortini, A., J. Physique 32 (1971) 895.

[16] London, F., « Superfluids » (Dover Public. Inc. N. Y.), 1950, Vol. I. 